\title{
Miranda
}

Revue pluridisciplinaire du monde anglophone /

Multidisciplinary peer-reviewed journal on the English-

speaking world

15 | 2017

Lolita at 60 / Staging American Bodies

\section{Hélène Machinal, Gilles Ménégaldo, Jean-Pierre Naugrette, Sherlock Holmes, un nouveau limier pour le $\mathrm{XXI}$ siècle.}

\section{Sylvie Crinquand}

\section{(2) OpenEdition Journals}

\section{Édition électronique}

URL : http://journals.openedition.org/miranda/10821

DOI : 10.4000/miranda. 10821

ISSN : 2108-6559

\section{Éditeur}

Université Toulouse - Jean Jaurès

\section{Référence électronique}

Sylvie Crinquand, « Hélène Machinal, Gilles Ménégaldo, Jean-Pierre Naugrette, Sherlock Holmes, un nouveau limier pour le XXIe siècle. », Miranda [En ligne], 15 | 2017, mis en ligne le 20 septembre 2017, consulté le 16 février 2021. URL : http://journals.openedition.org/miranda/10821 ; DOI : https:// doi.org/10.4000/miranda.10821

Ce document a été généré automatiquement le 16 février 2021.

\section{cc)}

Miranda is licensed under a Creative Commons Attribution-NonCommercial-NoDerivatives 4.0

International License. 


\title{
Hélène Machinal, Gilles Ménégaldo, Jean-Pierre Naugrette, Sherlock Holmes, un nouveau limier pour le XXI siècle.
}

\author{
Sylvie Crinquand
}

\section{RÉFÉRENCE}

Machinal, Hélène, Gilles Ménégaldo, Jean-Pierre Naugrette, Sherlock Holmes, un nouveau limier pour le XXI e siècle (Rennes : PUR, 201, 325 p, ISBN : 978- 2-7535-5144-2

1 Cet ouvrage passionnant regroupe des communications issues du colloque de Cerisy consacré à Sherlock Holmes et plus précisément au devenir du personnage au XXI ${ }^{e}$ siècle. Il s'articule en cinq volets suivis d'une annexe, d'une bibliographie générale, fort utile, et d'un index, et propose un voyage à travers plusieurs époques, plusieurs pays et plusieurs média, en gardant à l'esprit, telle une boussole, le personnage d'origine créé par Conan Doyle, et cet alliage de singularité et de plasticité qui lui permet de survivre aujourd'hui sous des formes très diverses. La diversité des supports analysés dans ce volume montre en effet combien le détective anglais est toujours populaire aujourd'hui, dans tous les sens du terme.

2 Hélène Machinal introduit le volume en revenant aux sources : celles qui ont conduit Doyle à créer son célèbre détective, mais aussi deux textes de Doyle, A Study in Scarlet et The Sign of Four, qu'elle considère comme fondateurs de l'œuvre à venir. Elle rappelle que la porosité entre réalité et fiction, qui conduit en partie à la création du mythe, et à l'illusion de faire face à un personnage réel demeurant bien 221B Baker Street, est instaurée dès les premiers textes par Doyle, qui inclut Dupin et Lecoq comme des personnages réels dans A Study in Scarlet. Cette introduction analyse ensuite l'évolution du personnage, depuis les premiers épisodes publiés dans le Strand Magazine, qui 
ancrent le récit dans une dynamique sérielle anticipant ainsi les actuelles séries télévisées, jusqu'aux plus récentes adaptations sous forme de bande dessinée ou de littérature jeunesse.

3 Un article liminaire de Jean-Pierre Naugrette fait suite à cette introduction générale, et se concentre sur la dimension matérielle des livres de Doyle, en s'appuyant sur le souvenir olfactif des livres de Poche par lesquels l'auteur de l'article fit connaissance avec Holmes, pour aborder le rapport entretenu par le détective avec la matérialité des documents qui lui sont confiés. Il analyse ensuite le passage du matériel au signe, aux codes que déchiffre Holmes avec son aisance coutumière, et souligne ainsi le rôle que joue l'intertextualité dans un texte-palimpseste, qui ancre le personnage dans un contexte fictionnel. Le grain de papier annoncé dans le titre de l'article est ainsi lu à un double niveau; d'abord comme signature, double elle aussi, celle de l'émetteur du message par le choix matériel du support de son message, mais aussi celle de la trace laissée par l'intertexte. Mais l'article suggère également une autre vision du grain de papier, liée au code, et dépeint un Holmes « hacker avant la lettre » (p. 36), capable des décryptages les plus audacieux, dont le terrain de jeu n'est pas tant le palimpseste que la toile, ce qui place ce détective lecteur scripteur au cœur des problématiques du XXI ${ }^{\mathrm{e}}$ siècle.

Ces fondations solides ayant été posées, la première partie de l'ouvrage se consacre aux origines du mythe et au Holmes du Canon, avec tout d'abord une analyse de l'influence de Wilkie Collins sur le personnage de Holmes. Mariaconcetta Costantini se place dans le cadre de l'histoire littéraire pour rappeler la critique de Graham Greene selon laquelle The Sign of Four doit son intrigue à celle de The Moonstone, et analyse en détail les parallèles entre Holmes et le sergent Cuff, mis en scène dans le roman de Collins, ainsi que les points sur lesquels Doyle s'éloigne de son prédécesseur. Elle en conclut que Collins propose une écriture plus audacieuse, plus avant-gardiste que son successeur. Ce recadrage historique cède à la place à une perspective narratologique, et Nathalie Jaëck défend l'idée selon laquelle les intrigues holmésiennes créent une addiction, à cause de laquelle la fin ne suffit jamais, parce que la fin est toujours conçue comme avant-dernier épisode. Son analyse se fonde sur une étude rapprochée des dernières pages des nouvelles, où elle montre que celles-ci se partagent entre un sentiment d'anticipation (de la nouvelle suivante) et de frustration (devant des révélations incomplètes). Enfin la première partie se conclut par une analyse plus thématique, avec l'article de Dominique Meyer-Bolzinger consacré à la présence de l'imaginaire de l'autopsie dans le texte. Elle souligne pour commencer le fait que les autopsies semblent absentes des textes de Doyle, le mot lui-même n'étant jamais utilisé, pour ensuite montrer comment l'autopsie devient métaphorique, puisqu'il s'agit « de découper le monde, les corps, les textes, pour les transformer en récits. » (p. 87)

La seconde partie de l'ouvrage est consacrée à une première série de variations, textuelles, et se compose de deux articles complémentaires. Christian Chelebourg prend pour objet d'étude la littérature jeunesse, et plus particulièrement les romans de Nancy Springer publiés au tout début du XXI è siècle, où la mise en scène de la sœur du détective, Enola Holmes, permet à ses jeunes lectrices de repérer «les indices de la domination masculine ", et répond ainsi à la vision sexiste de Doyle. Lauric Guillaud s'intéresse pour sa part aux pastiches de Holmes, et plus précisément à un détective américain, Solar Pons, créé par August Derlech en 1928, pour réfléchir à la manière dont le pastiche peut se révéler original pour offrir une nouvelle forme de création 
littéraire. Il analyse le difficile équilibre entre imitation et subversion du modèle, " entre fidélité et volonté d'autonomie » (p.128) pour conclure que Derlech a au moins réussi à faire entrer son détective dans la culture populaire, puisqu'un site web lui est consacré.

6 La troisième partie de l'ouvrage, intitulée "Variations sérielles ", et consacrée aux séries télévisées, s'ouvre sur un article d'Hélène Machinal qui traite de la série anglaise Sherlock. Elle propose une analyse fine et rapprochée de la série dans ses liens aux nouvelles d'origine, pour montrer comment cette adaptation utilise l'original tout en proposant un changement de paradigme, faisant ainsi entrer le texte holmésien dans le $\mathrm{XXI}^{\mathrm{e}}$ siècle. La même série télévisée est ensuite revisitée par Camille Fort, qui met l'accent sur la représentation de Londres mise à l'écran, toujours en conservant les écrits de Doyle en point de référence ; elle commence par réfléchir à ce qu'elle nomme la «fonction-Londres» (p. 160) en clin d'œil à Foucault pour terminer avec Michel de Certeau et la distinction entre le lieu et l'espace. Enfin Maud Desmet élargit le corpus aux séries télévisées les plus récentes, pour montrer comment l'influence de Holmes est perceptible dans les fictions les plus contemporaines sous les traits d'un " personnage archétypal d'enquêteur » dont elle analyse les caractéristiques à la lumière du corpus holmésien.

7 Le quatrième volet se tourne vers les adaptations cinématographiques, avec un premier article dans lequel olivier Cotte s'intéresse à la figure de Watson et recense les modes de représentation de l'ami du détective, en allant du «Watson occulté» aux " variations Watson », et pour terminer non sans humour avec le « Watson (enfin) plus intelligent que Holmes». Laura Marcus poursuit l'analyse avec des références au domaine germanique, en mettant l'accent sur un film autrichien comique, The Man who was Sherlock Holmes, qu'elle introduit par une réflexion sur ce qui, chez Holmes, semble appeler l'imitation et la parodie. Gilles Menegaldo se tourne vers la rencontre entre Holmes et Jack the Ripper, mise en scène dans plusieurs films, dont trois sont plus précisément convoqués dans son étude qui démontre comment la plasticité de ces deux figures mythiques facilite leur rencontre. Enfin Julien Guieu analyse lui aussi une rencontre, qui peut sembler plus improbable à première vue, entre Holmes et Don Juan dans le film Broken Flowers de Jim Jarmusch, pour en conclure que «s'il y a des Don Juan, il n'y a qu'un seul Sherlock » (p. 250).

8 La dernière partie de l'ouvrage, intitulée "Variations transmédiatiques », conclut en proposant deux cas spécifiques de transmédialité, le théâtre et la bande dessinée. Caroline Renouard s'intéresse aux mises en scène du personnage en France, à partir de l'adaptation française de la pièce de William Gillette, et montre que ces adaptations sont partagées entre fidélité à l'original et trouvailles novatrices. Et Jean-Paul Meyer poursuit l'analyse sur la bande dessinée, en mettant l'accent sur deux séries, Holmes et Les Quatre de Baker Street, pour montrer en quoi la spécificité du genre permet une adaptation renouvelée et originale. Enfin, en annexe, Antoine Faivre propose une filmographie commentée des films muets avec Holmes.

9 L'ouvrage propose ainsi un tour d'horizon très complet des tribulations de Holmes à l'époque contemporaine, tour d'horizon qui se double d'analyses stimulantes, qui conduisent à réévaluer la figure du détective, par le mouvement constant de va-etvient entre l'original et les adaptations. 
INDEX

Mots-clés : Doyle, Holmes, roman policier, détective, cinéma, réécriture, adaptation

Keywords : Doyle, Holmes, detective fiction, detective, cinema, rewriting, adaptation

\section{AUTEURS}

\section{SYLVIE CRINQUAND}

Professeure des Universités

Université de Bourgogne-Franche-Comté,

sylvie.crinquand@u-bourgogne.fr 